\title{
DIFFERENTIAL ROTATION AND GIANT CELL CIRCULATION OF THE SOLAR $\mathrm{Ca}^{+}$-NETWORK
}

E. H. SCHRÖTER and H. WÖHL

University Observatory Göttingen, West Germany

High precision computer controlled tracings of bright $\mathrm{Ca}^{+}$-mottles were performed during 1974 and 1975 at the Locarno Observatory of Göttingen to study solar differential rotation and to search for giant cell circulation pattern. Details of the observing method and the results from observations during 1974 have been published very recently (Schröter and Wöhl, 1975). Our method consists of measuring the position of 5-15 bright $\mathrm{Ca}^{+}$-mottles with respect to the center of the solar disc
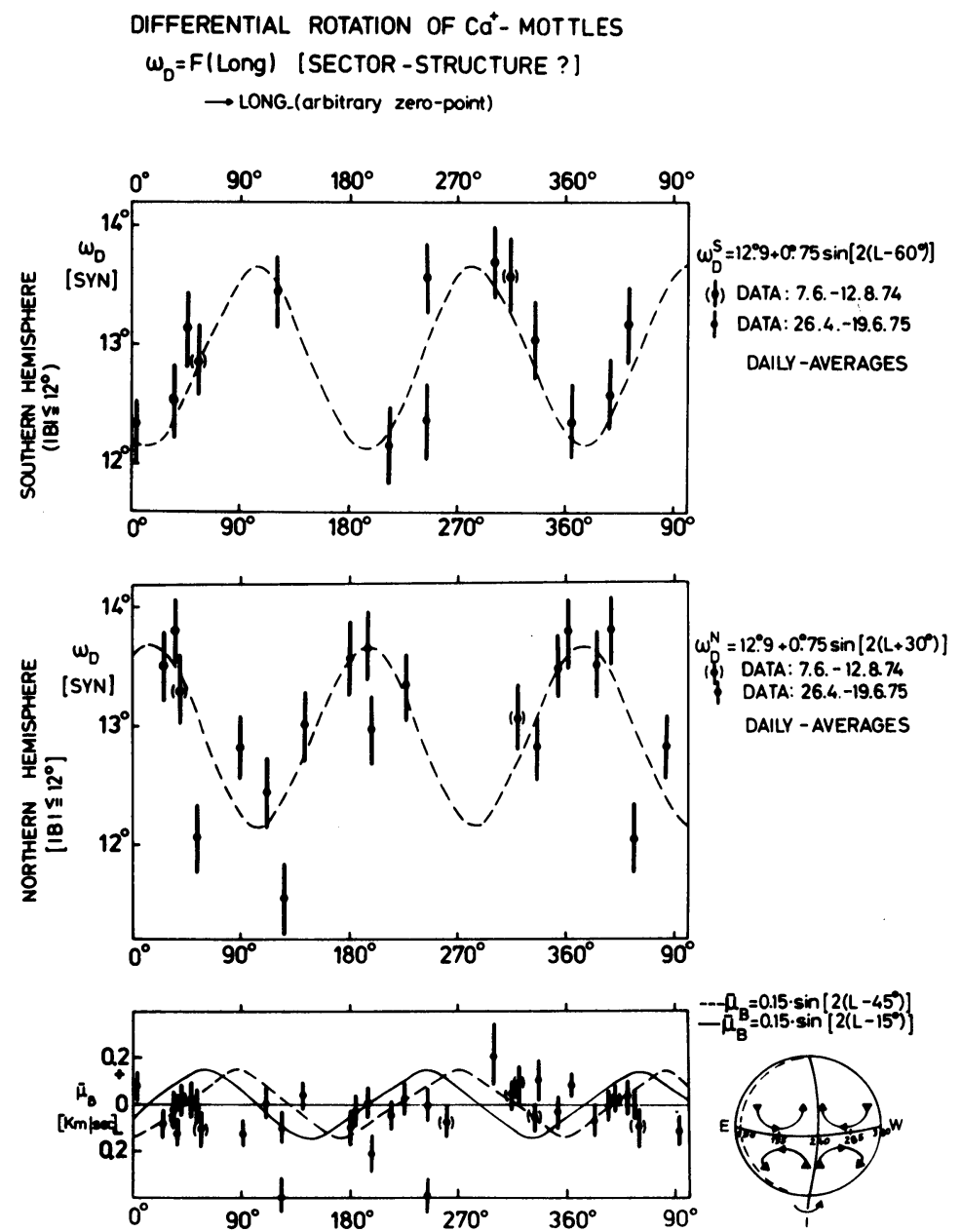

Fig. 1. 
every 10 to 15 min during 4 h every day. A computer controlled program determines first the center of the disc and immediately afterwards the right ascension and declination of a $\mathrm{Ca}^{+}$-mottle which was centered by the observer into a $1^{\prime \prime}$-diameter spectrograph pinhole by watching a TV-monitor displaying a $\mathrm{Ca}^{+}-\mathrm{K}$-line solar image. From a linear least squares fit of the observed positions the solar latitude and longitude were computed for the beginning and the end of the daily $4 \mathrm{~h}$ observation period. From this the components in latitude and longitude of the proper motions were derived which result from the differential rotation, possible giant cell circulation and the small scale random walk of these features.

The main goal of the 1975 observing program was to search for a sector-like structure of the solar rotation within the equator belt as a result of giant cell circulation pattern. We traced $341 \mathrm{Ca}^{+}$-mottles in latitudes $\leqq 12^{\circ}$ from 1975 , April 26 to June 19. The result of these observations are shown in Figure 1, where the synodic rotation angle per day $\left(\omega_{\mathrm{D}}\right)$ is plotted as a function of the solar longitude for the northern and southern hemisphere separately. The points represent the average longitudinal motion component from the 5-15 bright mottles traced during one day. The length of the bars corresponds to the mean of the daily rms deviation. These rms deviations do not present the accuracy of measurements which is much better, but reflect mainly the random walk of the $\mathrm{Ca}^{+}$-mottles superposed on the drift, due to rotation. Clearly, a straight line representation of the data, resulting in a longitude independent rotation rate, is rather unsatisfactory. A much better fit is obtained by a sinoidal-curve leading to two sectors with faster than average rotation and two sectors with slower than average rotation for each hemisphere. The deviation of 0.75 day $^{-1}$ corresponds to $\Delta v=0.105 \mathrm{~km} \mathrm{~s}^{-1}$. There is a $90^{\circ}$ phase difference between the sectors of the northern and southern hemisphere. These results suggest

\section{SECTOR - STRUCTURE IN SOLAR ROTATION ?}

TIME: $2.0369-2.3591$ in H.H.time scale ( 4.5 rotations)

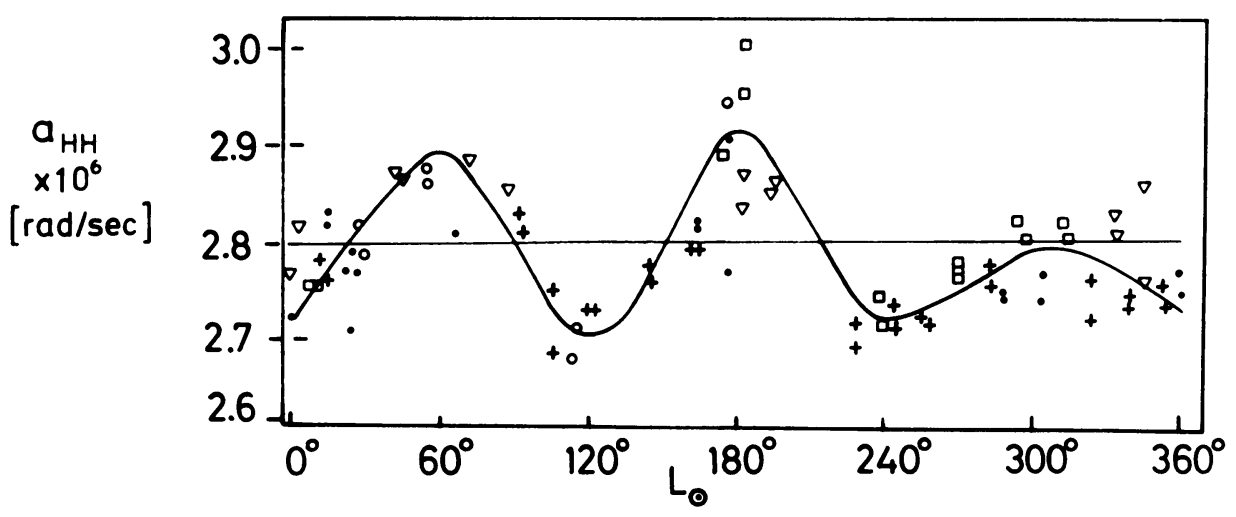

Howard and Harvey parameter $\underline{a}$ as function of longitude

Fig. 2 . 
very much a giant cell circulation pattern as indicated at the bottom of Figure 1 . This coarse model may well be tested by the behavior of the latitudinal component of the proper motions. In longitudes $L=60^{\circ}$ and $240^{\circ}$ a poleward (equatorward) drift is to be expected in the northern (southern) hemisphere whereas drifts with opposite directions should occur at longitudes $L=150^{\circ}$ and $330^{\circ}$. We, therefore, converted the sign of the latitudinal motion component $\mu_{B}$ in the southern hemisphere and plotted $\mu_{B}$ from the northern and southern hemisphere as a function of the longitude $\left(\mu_{B}>0\right.$ means a poleward drift, units: $\left.\mathrm{km} \mathrm{s}^{-1}\right)$. The full-line curve corresponds to the circulation pattern model as indicated at the right, the dashed-line curve is a somewhat better fit of the data. However, both representations are not very convincing. We have to wait for the final reduction using more data (partly already obtained but not yet reduced) to check whether such a simple model of a giant cell circulation pattern is capable of representing well all observations.

Being aware of the fact that such a pattern, if existing, should also have been observed by previous measurements, we performed some sample probe compilations with the data published by Howard and Harvey (1970). We found several time periods in their Table II where the speed of the equator rotation clearly varied periodically for 2-4 solar rotations. For the sake of space we present here only one example. Figure 2 shows the equatorial rotation speed $a_{\mathrm{HH}}$ (units: radians per second) from Table II of Howard's and Harvey's paper as function of the solar latitude for the time interval 1967, January 14 to April 15. The different symbols represent successive solar rotations. This time, a 6-sector structure of roughly $60^{\circ}$ extension in longitude is indicated with no phase difference between the northern and southern hemisphere. The amplitude of deviations is of the same order of magnitude as for our observations. However, Howard and Harvey reduced their data in a fashion being rather unsuitable for the detection of such sector structures, since full disc (or full hemisphere) observations were used to derive the rotation rate of the equator zone. Hence, the fluctuation of $a_{\mathrm{HH}}$ in Figure 2 is a kind of a net effect resulting from an uncomplete smoothing of the sector structure by the reduction procedure of Howard and Harvey.

After having reduced in final form all available data we shall submit a more detailed presentation to Solar Physics.

\section{Acknowledgement}

We thank Mr D. Soltau for the help in numerical reductions. The Locarno Solar Observatory is operated by the Deutsche Forschungsgemeinschaft.

\section{References}




\section{DISCUSSION}

Stenflo: There seems to be some contradiction between your results and those presented by Howard in his review, which showed coherent, simultaneous changes in the rotation velocity in the northern and southern hemispheres. Would you like to comment on this?

Schröter: I forgot to mention this point. Yes, what puzzles me, is the fact that during our observational period 1975, April 24-June 19, the large scale circulation cells of the northern hemisphere showed a circulation sense opposite to that of the southern hemisphere, whereas in those periods I picked out from Howard's and Harvey's Table II, apparently the cells in both hemispheres showed the same sense of circulation. At the moment I can only guess the reasons for this discrepancy: (a) Howard's and Harvey's data we used, have already been reduced according to a certain procedure and it is very hard to overlook to what extent such a large scale circulation pattern has been smoothed out by this procedure. One should look into the original data and reduce them in a more problem-oriented way. (b) Our observations cover a single period of 2.5 rotations only. We cannot exclude that such an antisymmetric mode of large scale circulation is not a common but a rare phenomenon occurring from time to time only. We are continuing our programme and shall be able to answer this question more definitely at the end of this year.

Howard: We plan to rereduce all the older data with the new reduction technique described in our previous paper. I would like to point out that for the two days from last summer for which we showed results in the previous paper, the southern hemisphere rotated slightly faster than the northern hemisphere. These data were from last summer (1974).

Schröter: We found in the period 1974, June-August 12, the southern equator belt to rotate faster than the northern belt in agreement with your findings. However, in comparing both results one should not forget, that our results refer to $\mathrm{Ca}^{+}$-chromospheric layers.

Gilman: (1) Please draw your postulated circulation pattern on the board.

(2) Comment: This is the opposite of the dominant symmetry suggested by non-axisymmetric convection theory.

Schröter: To (1): I did so. (2) Since our observations cover a single period of 2.5 solar rotation only this type of large scale circulation may not necessarily be a common, but rather a rare phenomenon. But there is no doubt that the opposite sense of circulation in both hemispheres is far out of observational errors. 